\title{
High-throughput identification of chrysanthemum gene function and expression: An overview and an effective proposition
}

\author{
Toan Khac Nguyen $\cdot$ Jin Hee Lim
}

Received: 4 July 2021 / Revised: 28 July 2021 / Accepted: 28 July 2021

(c) Korean Society for Plant Biotechnology

\begin{abstract}
Since whole-genome duplication (WGD) of diploid Chrysanthemum nankingense and de novo assembly whole-genome of $C$. seticuspe have been obtained, they have afforded to perceive the diversity evolution and gene discovery in the improved investigation of chrysanthemum breeding. The robust tools of high-throughput identification and analysis of gene function and expression produce their vast importance in chrysanthemum genomics. However, the gigantic genome size and heterozygosity are also mentioned as the major obstacles preventing the chrysanthemum breeding practices and functional genomics analysis. Nonetheless, some of technological contemporaries provide scientific efficient and promising solutions to diminish the drawbacks and investigate the high proficient methods for generous phenotyping data obtaining and system progress in future perspectives. This review provides valuable strategies for a broad overview about the high-throughput identification, and molecular analysis of gene function and expression in chrysanthemum. We also contribute the efficient proposition about specific protocols for considering chrysanthemum genes. In further perspective, the proper high-throughput identification will continue to advance rapidly and advertise the next generation in chrysanthemum breeding.
\end{abstract}

Keywords Chrysanthemumbreeding, Gene expression, Gene function, Genomics analysis, High-throughput sequencing, Phenotyping data

\section{Introduction}

High-throughput sequencing methods are recognized as

T. K. Nguyen · J. H. Lim $(\bowtie)$

Department of Plant Biotechnology, Sejong University, Seoul 05006, Korea

e-mail: jinheelim@sejong.ac.kr essential innovation tools for quantitative sequencing nucleic acid molecules. High-throughput sequencing techniques (HST) are now reducing price, replacing the analysis of traditional cloning library, and probably identifying techniques in the adjacent future (Huse et al. 2008). Based on the read-length achievement, HST accesses an increase in coverage read-depth to identify phylotypes, to estimate diversity, and discover metagenomic characterization of genetic complex traits.

Chrysanthemum, which is an important crop of the Asteraceae family, is high-value in floricultural crops and is stood the second in the florist market trade throughout the world (Nguyen and Lim 2019; Nguyen et al. 2020). Chrysanthemums were used for medicinal purposes as the birth-place in China where they were first cultivated for traditional medicine treatments (Nguyen and Lim 2019). They are characterized by types, flower shapes, and their colors are made as high value in floral crop (Nguyen and Lim 2019; Nguyen et al. 2020).

Despite the challenges conferred by global climate change and the immediately burgeoning human population, techniques to achieve the giant production and high quality chrysanthemum with reducing input are critically required (Tester and Langridge 2010). Therefore, relevant approaches for progressing the best chrysanthemum production that would obtain disease and insect resistances, various petal colors, shapes, and many type of flowers, has been recommended (Teixeira da Silva et al. 2013).

Additionally, the lack of genomic chrysanthemum data has been solved by various achievements to study deeply in chrysanthemum genomic functional with high-throughput sequencing tools ( $\mathrm{Su}$ et al. 2019). The improvement of functional genomic and genetic techniques is over three decades, principally technological sequencing tool. In $C$. nankingense, the WGD of diploid level have been sequenced (Song et al. 2018), and the functional investigation of the chrysanthemum genome has accessed the high-throughput 
Table 1 Chrysanthemum key genes and their functions

\begin{tabular}{|c|c|c|}
\hline Name & Function & References \\
\hline CmHSFA4 & $\mathrm{CmHSFA4}$ (in overexpression) improved the salt tolerance of chrysanthemum & (Li et al. 2018) \\
\hline $\begin{array}{l}\text { WRKY transcription } \\
\text { factor }\end{array}$ & $\begin{array}{l}\text { correlated in stress responses including salt stress, drought, disease, aphid } \\
\text { resistance }\end{array}$ & $\begin{array}{l}\text { (Fan et al. 2015; Li et al. } \\
\text { 2015a; Li et al. 2015b; Fan et } \\
\text { al. 2016; Jaffar et al. 2016; } \\
\text { Liang et al. 2017; Wang et } \\
\text { al. 2017) }\end{array}$ \\
\hline CmDREB6 & CmDREB6 (in overexpression) improved the heat tolerance & (Du et al. 2018) \\
\hline CmCPL1 & $\begin{array}{l}C m C P L 1 \text { increased (in overexpression) or decreased (in knockdown) the } \\
\text { heat tolerance }\end{array}$ & (Qi et al. 2018) \\
\hline$C l C B F 1$ & $\begin{array}{l}C l C B F 1 \text { (in overexpression) improved its drought tolerance and salt stress } \\
\text { in C. lavandulifolium 'White Snow' }\end{array}$ & (Gao et al. 2018b) \\
\hline $\mathrm{CmCYC2}$ & $\begin{array}{l}\mathrm{CmCYC2c} \text { increased (in overexpression) flower number and ray floret } \\
\text { length, deficient to completely modify flower shape }\end{array}$ & (Huang et al. 2016) \\
\hline CmTCP20 & obligation for petal elongation growth & (Wang et al. 2019a) \\
\hline CmANRI & encouragement nonessential root and lateral root development & (Sun et al. 2018) \\
\hline CmERF053 & $\begin{array}{l}\text { maintaining the new regulating shoot function and developing lateral root, } \\
\text { affecting drought resistance }\end{array}$ & (Nie et al. 2018) \\
\hline$C m-B B X 24$ & $\begin{array}{l}\text { transgenic RNA interference }(C m-B B X 24-R N A i) \text { suppressed flowering } \\
\text { earlier } \sim 20 \text { days than wild type }\end{array}$ & (Yang et al. 2014) \\
\hline$C m N F-Y B 8$ & CmNF-YB8 expression with cmo-MIR156 influences flowering time & (Wei et al. 2017) \\
\hline$C t A 3{ }^{\prime} 5^{\prime} G T$ & $\begin{array}{l}C t A 35^{\prime} G T \text { (true blue color), encoding UDP-glucose: Clitoria ternatea with } \\
\text { anthocyanin } 3^{\prime}, 5^{\prime} \text {-O-glucosyltransferase, and Campanula medium with } \\
\text { CamF3 } 5 \text { H, encoding F3' } 5^{\prime} \mathrm{H}\end{array}$ & (Noda et al. 2017) \\
\hline
\end{tabular}

platform (Yagi 2018). In C. seticuspe, de novo assembly was studied in whole genome (Hirakawa et al. 2019). Some of chrysanthemum key genes were characterized their functions such as stress tolerance (abiotic and biotic), plant growth and development, flowering time, and flower color (Table 1).

High-throughput sequencing techniques have been enabled by increased the new knowledge of modern plant genomes (Furbank 2009). The applicable genomic data have been obtained with accommodate technologies to be enable to identify the functional of plant genes such as photonics applications (Yeong et al. 2019), functional plant biology (Poorter et al. 2012), phenotyping computers vision-based (Mochida et al. 2018), and robotics (Coppens et al. 2017). Thus, they should be expanded to allow chrysanthemum physiology and phenomics to improve correlation of chrysanthemum genomics.

Here, we summarize the emerging methods in chrysanthemum species and highlight the technological biases of high-throughput sequencing technologies. Next, we focus on the ongoing applications in chrysanthemum phenomics, and note the efficient proposition in high-throughput sequencing based on research design. We conceive the major challenges in chrysanthemum phenomics.

\section{High-throughput sequencing methods for Chrysanthemum species identification}

High-throughput techniques could be carried quantification PCR, hybridization-based (microarrays), second-generation fingerprinting (RADseq), and sequence-based (meta-barcoding, meta-transcriptomics, meta-genomics).

In 2005, PCR quantification of phytoplasma DNA in $C$. carinatum and insect (Macrosteles quadripunctulatus) host was described. Primers and a TaqMan probe were designed on a target cloned specific ribosomal chrysanthemum fragment. They were also applied on ITS1 (internal transcribed sequences) and $18 \mathrm{~S}$ rDNA (plant) for amplification from $C$. carinatum and M. quadripunculatus (Marzachì and Bosco 2005). Chrysanthemum yellows phytoplasma (CYP) were found a few hundred thousand in the highest amounts in leaves and roots (Saracco et al. 2005). CYP concerning young developing leaves and roots had been indicated (Saracco et al. 2005).

The Asteraceae introduces various polyploid species, and broad crossing in the proceeding of hybridization by genetic and epigenetic alterations. In Asteraceae, the consequences of hybridization were characterized in the large genomic, transcriptomic and epigenomic. These adjustments conducted 
the processing hybridization in the cross-sections $C$. nankingense $\times$ Tanacetum vulgare and $C$. crassum $\times$ Crossostephium chinense. These cytosine methylation changes could be introduced the evolutionary processes of the mechanisms newly formed intergeneric hybrids formation (Wang et al. 2013a). The paired-end sequencing presented over 53 million reads among 81 amplicon markers, 20 polymorphic markers, and 100 random primer pairs from $C$. nankingense mRNA in Illumina platform (Wang et al. 2013b).

RNA-Seq technology was applied to chrysanthemum transcriptomic which was responded to Alternaria tenuissima inoculation by the Illumina HiSeq ${ }^{\mathrm{TM}} 2000$ platform. Twenty three gene selections were identified on the profiled transcription by validating the RNA-Seq output using qRT-PCR (Li et al. 2014). The chrysanthemum embryo including 1037 miRNA target genes was characterized by transcriptome data and 228 miRNAs were validated with symbolic changes in frequency expression through embryotic development (Zhang et al. 2015). The miRNAs response was discovered to lownitrogen stress in $C$. nankingense which the results presented overlapping or effecting unique response to nitrate stress in leaves and roots, including miR156, miR169, and miR393 (Song et al. 2015).

Quantitative real-time PCR (qPCR) uses to identify the stage of flower development, which is an identified 9 candidate reference genes for their expression through $C$. morifolium and C. lavandulifolium samples. SAND (SAND family protein) was most thoroughly expressed in all or different $C$. lavandulifolium tissues. Both SAND and PGK (phosphoglycerate kinase) achieved the most stable in large flower, and the best $P G K$ was presented in pot chrysanthemums (Qi et al. 2016). Based on the use of 454 sequencing platform, the $C$. indicum completed chloroplast genome was achieved with $150,972 \mathrm{bp}$ in length including inverted repeats (IR; 24,956 bp), large single copy (LSC; 82,741 bp), and small single (SSC; 18,319 bp) regions (Xia et al. 2016). miRNAs target genes were studied on the normal and abnormal embryos within three sRNA libraries by RNA-Seq to identify 170 miRNAs with 41 special miRNAs in the paternal chromosome doubling cross including miR169b, miR440, and miR528-5p (Zhang et al. 2017). For floral traits of chrysanthemum, the 454-pyrosequencing technology was used to construct normalized cDNA libraries in chrysanthemum. Chrysanthemum libraries presented $\sim 3,77$ million high-quality reads through assembling into $\sim 213,000$ contigs (Sasaki et al. 2017).

Oxford Nanopore long-read-technology was used to sequencing $C$. nankingense genome, its genome evolution was consumed by repetitive element expansion bursts, and the
WGD chrysanthemum classified near WGD sunflower, which deviated from chrysanthemum $\sim 38.8$ million years ago (Song et al. 2018). The diploid $C$. seticuspe was studied as a model plant in the cultivated chrysanthemum and analyzed de novo whole-genome assembly by Illumina platform. The results showed that the assembled sequences were $2.72 \mathrm{~Gb} ; 354,212$ consisting scaffolds; the $3.06 \mathrm{~Gb}$ of $C$. seticuspe genome was covered to $89.0 \%$ estimated by k-mer analysis (Hirakawa et al. 2019).

\section{Chrysanthemum gene functions and expressions}

MADS-box transcription factors were designed in functionally characterized in C. grandiflorum such as MADS (CDM) 8, CDM41, CDM111, and CDM44. CDM44 protein combines to $C D M$ proteins which are constructed to the $A P 1 / F U L$ and $A G$ subfamilies, and made the expression in larger than order heterodimer complex with B-type $C D M$ proteins in C. grandiflorum (Shchennikova et al. 2004).

CSFTL3 had found early induced flowering time in non-inductive conditions in $C$. seticuspe accessions. The expression levels of CsFTL3 were higher in its leaves, therefore, the CSFTL3 gene product is one of the key regulators in photoperiodic flowering time (Oda et al. 2012). In the study of cultivated chrysanthemum breeding, the salt stress can be arranged valuable information for describing the salt tolerance mechanism in plant. In $C$. lavandulifolium, cDNA amplified fragment length polymorphism (cDNA-AFLP) was analyzed to identify the differential gene expression in chrysanthemum leaves in $\mathrm{NaCl}$ response. By using cDNA clone and BLASTx program, 25 expressed sequence tags (ESTs) were identical to known functional genes (He et al. 2012).

The systemic flowering inducers (florigens) and inhibitors (antiflorigenes) can be changed in the day length in leaves that regulated to floral initiation in shoot. The Anti-florigenic FT/TFL1 family protein (AFT) was studied in C. seticuspe. $C S A F T$ induction was produced by phytochrome signals; and flowering step was developed in the photosensitive phase (Higuchi et al. 2013). In C. morifolium 'Fall Color', the study of regulated hormone biosynthesis and signaling was performed in two cDNA libraries to identify 8,558 unique transcripts in responsive dehydration, containing 307 transcription factors, and 229 protein kinases by Illumina technology (Xu et al. 2013).

The C. lavandulifolium transcriptome was shown 108,737 unigenes high-quality ( $\sim$ GB data), 58,093 proteins sequencing, and 211 flowering-related unigenes by using 
Illumina HiSeqTM 2000 sequencing platform. Moreover, the genes expression analysis identified 14,406 unigenes related to the visible bud form and seedling stage. In the transcriptome of $C$. lavandulifolium, many unigenes with high correspondence to important genes that are complicated in the gibberellin, photoperiod, autonomous pathway, and vernalization were analyzed, including FLOWERING LOCUS C (FLC), CONSTANS (CO), FLOWERING LOCUS T (FT), and SUPPRESSOR OF OVEREXPRESSION OF CONSTANS 1 (SOC1) (Wang et al. 2014).

The function of an MYB transcription factor (especially focuses on RsMYB1 ectopic expression isolated from radish Raphanus sativa) was identified $C m F 3 H, C m D F R$, and CmANS biosynthetic key genes which are responsible for anthocyanin products in transgenic chrysanthemum and are in possession a negative feedback to non-transgenic line. Especially, RsMYB1 was not found any change in morphological characteristics, such as number of flower, leaf size and shape, and plant height in chrysanthemum cv. 'Shinma' (Naing et al. 2015). The Illumina platforms were performed to sequence chrysanthemum buds (from vegetative buds to floral buds) in C. morifolium. 91,367 unigenes were identified in $15.4 \mathrm{~Gb}$ of reads, including 43,137 unigenes showed similarity protein. DEGs expressed genes (1,876 DEGs including 1,516 up- and 360 down-regulated) were analyzed between floral buds and vegetative buds, and 3,300 DEGs (1,277 up- and 1,706 down-regulated) were scanned between floral buds and buds (Liu et al. 2015).

qPCR was identified reference genes in the developmental flower stage in C. morifolium and C. lavandulifolium. The suggestion of good candidate genes was appraised and validated by four algorithms such as Bestkeeper, NormFinder, GeNorm, and Ref-finder. In C. lavandulifolium, all samples were found the most strongly expressed genes in $S A N D$ family protein. The study was screened on both $S A N D$ and $P G K$ (phosphoglycerate kinase) to achieve the most constant in Chinese chrysanthemum cultivars with large flower, and the potted chrysanthemums was the best in $P G K$ (Qi et al. 2016). The SQUAMOSA promoter binding protein-like (SBP or $S P L$ ) family is defined to know as functional transcription factors by a plant-specific DNAbinding domain. Twelve SBP-like (SPL) genes were shown six of these genes included a miR156 target site, and five CmSPLs were targeted by miR157 in C. morifolium. Furthermore, $50 R L M-R A C E$ were mapped to the cleavage sites in CmSPL2 and CmSPL3 (Song et al. 2016b). The analyses of 20 DNA binding with one finger $(D O F)$ genes were shown that $13 \mathrm{CmDOFs}$ could be focused by 16 miRNA families in C. morifolium based on transcriptomic sequences. These genes were expressed patterns of 6 pairs of paralogous $C m D O F$ genes which were discovered to effectively differ from one another, except for $\mathrm{CmDOF} 6$ and CmDOF7 (Song et al. 2016a). The screening of MADS-box gene was performed on short vegetative phase (SVP) like genes from C. morifolium 'Jinbudiao' and constructed as CmSVP to consist 672 bp encoding 223 amino acids. CmSVP is conversely expressed in the shoot apex and leaves, and it is expressed in the decreasing concentration in both flower buds and flowers (Gao et al. 2017).

The isolated stem segments was performed to analyze bud outgrowth in auxin (IAA), strigolactone (GR24), and auxin transport inhibitor (NPA) to identify expression levels in auxin transport (CmPIN1) and signaling (CmAXR2), bud dormancy $(C m B R C l, C m D R M I)$ and strigolactone biosynthesis (CmMAXI, CmMAX3). The expression of CmBRCl and $C m D R M l$ was decreased and the expression of CmPINI was increased in the control of high dividing cell percentage in bud outgrowth. The inhibition by IAA and IAA and GR24 concurred with low dividing cell percentage and unaltered or increased expressions of $\mathrm{CmBRCl}, \mathrm{CmDRM1}$, and CmPIN1. Notwithstanding, GR24 treatment indicated reduced bud outgrowth that was prevented by NPA (Dierck et al. 2018). The study of anthocyanin biosynthesisrelated genes CmplCHI, CmplaNS, CmplCHS, CmplF3H, and CmplDFR was performed in C. morifolium 'Pelican'. Two genes, CmplCHSs and CmplCHI, indicated that a high gene expression level from stage 1 to show un-pigmented petals. Two genes (CmDFRs and CmANS) performed in a high gene expression level with pale pink petals from stage 2. Notwithstanding, CmplDFR2 displayed constant gene expression from stage 2 to 6 , dissimilar the other isolated genes (Puangkrit et al. 2018). The analyses of 23 $C m G R A S$ genes in the GRAS gene family were performed to $C$. morifolium transcriptome. The CmGRAS4 and CmGRAS10 were powerfully transcribed in flowers and roots. In the other hand, the CmGRAS19 and CmGRAS20 was immersed in the reproductive tissues to be reactive to phytohormones and stresses (Gao et al. 2018a). A transcriptional regulator response to many abiotic stresses, such as RNAPII CTD phosphatase-like 1 ( CmCPLI), was isolated in C. morifolium. This analysis was performed 955 residue gene product containing the FCPH catalytic domain, one local nuclear signal, and two binding motifs in double-stranded RNA. The overexpression and knockdown of CmCPL1 was overexpressed and knock-downed to come out the result to increase and diminish the tolerance heat stress, which can be dependent on the regulation of CmCPL1 and on the expression of downstream responsive genes in chrysanthemum 
(Qi et al. 2018). RNA-Seq platform achieved a de novo transcriptome assembly to determine chrysanthemum (Dendranthema grandiflorum var. 'Jinba') transcription response to low temperature. By using Illumina technology, total 7583 differentially expressed genes (DEGs) of 36,462 annotated unigenes were discovered (Wang et al. 2018a). The highthroughput sequencing was used to analyze the genome-wide transcriptome of $C$. morifolium 'Chuju', which was wellknown as a famous traditional Chinese medicine species. Total 63,854 unigenes were obtained with an average length of $741 \mathrm{bp}$, and 12 unigenes complicated in flavonoid biosynthesis were commonly verified by using qPCR (Yue et al. 2018).

The functions of R2R3-MYB transcription factor (TF) genes are mainly associated to the accumulation of anthocyanins that have not been study well in chrysanthemum. By the use of phylogenetic and alignment analyses, some genes such as phenylpropanoid (CmMYB4 and CmMYB5), flavonoid repressor $(R 2 R 3-M Y B)$, anthocyanin biosynthesis activator (CmMYB6), flavonol biosynthesis regulator ( $C m M Y B 7)$, and negatively regulate anthocyanin biosynthesis (CmMYB4 and $C m M Y B 5$ ) were identified, while $C m M Y B 6$ and $C m M Y B 7$ were played a positive role (Hong et al. 2019).

A moderate domain spliced hairpin RNA (ihpRNA) is known as expression vector to intent DNA METHYLTRANSFERASE1 (CMMET1). Transgenic chrysanthemum materials (Zijingling) were consumed as root stock, or grafted onto Guoqinghong (GQH), and Huanshuijinqiu (HSJQ) non-transgenic the scions which were no signal in CmMETl gene, and presented phenotypes with early flowering. Methyltransferase gene expression was up-regulated to decreased expression (CMMET1), METHYLTRANSFERASE2 (CmDRM2), however, demethylating enzyme gene expression was down-regulated, DEMETER (CMDME), while the CHROMOMETHYLASEA (CmCMT3) expression level continued low and could be almost imponderable. Among the CmFT-likes genes that involve flowering time, CmFTL1 expression was downregulated, as well as CmFTL2 and CmFTL3 were up-regulated. The use of silencing CmMET1 could make less height, adjust phenotype, and improve earlier flowering ( $\mathrm{Li}$ et al. 2019). Four genes encoding pseudo-response regulator (PRR) related to flowering time were isolated from $C$. morifolium. The CmPRR2, CmPRR7, CmPRR37, and CmPRR73 genes are decidedly homologous to the counterparts of PRRs of Helianthus annuus (Wang et al. 2019b). The expression levels of six Chrysanthemum stunt viroid (CSVd) CSVd-siRNAs genes performed in cytokinin (CK), gibberellic acid (GA), and indole-3-acetic acid (IAA) biosynthesis transport. The growth of cell walls was down-regulated in infected plants, but mRNAs of those genes were not detected as possible target sequences of CSVd-siRNAs. On the different way, endogenous levels of abscisic acid, IAA, GA, CK, and the rate of IAA movement were not different between healthy and infected plants (Takino et al. 2019). A reference based on C. lavandulifolium transcriptome database was concerned the global changes in gene expression in $C$. lavandulifolium seedlings treated with $200 \mathrm{mM} \mathrm{NaCl}$ for 12 hours to compare with normal conditions. Total of 2254 DEGs (1418 up-regulated and 836 down-regulated genes) were discovered (He et al. 2019).

\section{Efficient propositions in high-throughput sequencing}

The Asteraceae family ( $\sim 24,000$ to $\sim 35,000$ species $)$ with gigantic genome sizes ( $\sim 3 \mathrm{Gbp}$ ) (Luo et al. 2017; Vallès et al. 2013) and $208 \mathrm{~kb}$ mitogenome sizes (Wang et al. 2018b). Based on your objective research goal for Chrysanthemum species, short-reads will not be respected for whole genome sequencing, but long-reads for the short-time sequencing is not an opportunity for the gigantic genome size such as Asteraceae (Nguyen and Lim 2019). Thus, chrysanthemum researchers should design their experiments based on their objective research. In chrysanthemum, miRNA genes play as gene expression in demanding regulator at infrequently transcriptional and post-transcriptional levels such as monitoring the transcriptional procedures, processing and maturation, control of accumulation levels, and verification of miRNA-target interactions based on the great achieved sequencing progresses. The finds on the great progresses must be achieved for high-throughput sequencing technology. These finds have been provided some original ways for the studies on genome-wide or transcriptome-wide.

Several HTS platform-based methods are considered to use for plant miRNA studies, such as ssRNA-seq (singlestranded RNA sequencing), dsRNA-seq (doublestranded RNA sequencing), degradome-seq (degradome sequencing), RNA-seq (RNA sequencing), sRNA-seq (small RNA sequencing), and RNA-PET-seq (paired end tag sequencing of RNAs). The innovative use of HST methods for inspected processing approaches of the miRNA precursors, recognition of the RNA rearranging sites on miRNA precursors, and discovery on the target interactions of novel miRNA species. The obliteration of the independent biological replicates seems to present in multiple spatially autocorrelated subsamples and vary recovered replicates. The origin of sample collection must be particularly consisted with essential hierarchical design and various level spatial autocorrelation. Pooling 


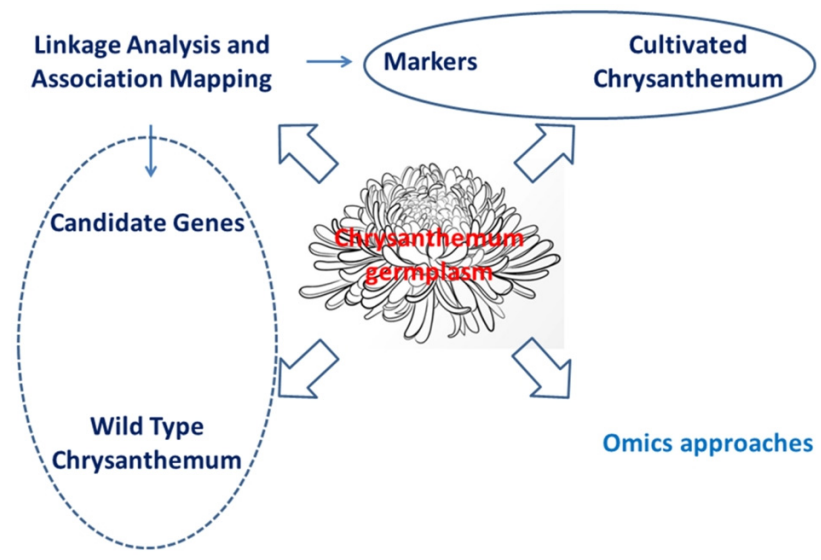

Fig. 1 Chrysanthemum germplasm studies. The circle shows the present study and the dashed circle shows the further studies in Chrysanthemum species

can be allowed vigorously examination cost, however, it is also diminished the small-scale resolution. However, the multiple testing independent samples are favored to the performance of approximate calculation in sampling error and number of spatiotemporal variability. Researchers should consider an extra replicate because it includes DNA in low-quality or restricted sequence reads from some samples (commonly $1 \% \sim 10 \%$ ). In the diversity study, the researchers must load more sample for univariate tests in the lower statistical power.

Based on the sensitive techniques to demolishing, external contamination and cross contamination; thus, HTS techniques will be required carefully collected samples, pre-treatment and handling inspect to avoid contamination and overrun by quick growing mildews or degradation of DNA or RNA. The pre-treatment steps, such as storage, drying with air or freeze, deep freezing, and preservation buffer fixing, were managed evenly fine for DNA. Dried, frozen, and deepfreezing DNA/RNA samples $\left(-80^{\circ} \mathrm{C}\right)$ would be considered for preservative potential analysis of DNA/RNA, fatty acids, and protein for further analyses. For considering contamination during all various analysis steps, it can be approved to set up the laboratory working space into moisten laboratory, PCR laboratory, and HTS preparation laboratory. The main authority of the contaminated HTS analyses is the different previous source of PCR steps, because of single strain of DNA molecule may be increasingly automatic amplified and sequenced. All the exteriors of RNA/DNA molecule can be control by efficient and economic cleaning of laboratory using DNase/RNase-containing solutions and UV-light. Negative controls should be considered in all stages of analyses because it can be detected mistakes and confirmed the track contamination.
Based on the work of HTS-based diversity analyses, the DNA/RNA markers should be clearly considered in the decision of taxonomic resolution. It is important to select the guiding methods for primers and choices of marker genes. For targeting specific host molecular, researchers should consider the primers that removing DNA host or adding blocking primers. Providing with $3^{\prime}$ nucleotideterminal modifications and oversupplied concentration, blocking primers inhibit annealing and make elongation of DNA host marker by exactly binding to DNA host downstream of regular primers. Based on sample molecular identification, forward and reverse primers should be tagged to enable multiplexing sequences. For Illumina sequence technique, 96 wells of DNA ligation can be combined by PCR. Identifiers tags are only cheaper and useful for sequencing analysis in their platform. In the ligation step, it is recommendable to reduce the tag amplicons because of starting with the use of two-base linker sequence in the same nucleotide with no match to any templates. To reduce the tag switching to minimize, the researchers have to consider strongly to identifier tags through both the reverse and forward primers.

For HTS analysis, the researchers should control the primer annealing temperature to benefit the amplification of sample templates which is with one or two mismatches to primers. On the other hands, the low input DNA template should be affected to the result of lower inhibitors and reduced chimeric sequences. Based on the stochastic variation, the researchers should be used at least two PCR replications that can be used for the next sequencing step; and it is defensed on the use of sequencing platform and further analyses. It is recommend that the use of specific adapter ligation and the choice of platform sequencing should be linked together to reduce the risk and the failure of service provider. To reduce the contamination and sequencing errors, the researchers should run three types of control sample in the same direction. If the experience has a few samples, the limited technical replication may be appropriate to estimate the method reproducibility and performance for upgrading protocols.

\section{Conclusion}

This study provides the overview in chrysanthemum molecular breeding and gene expression. To further investigation, the higher techniques should be upgraded for phenotyping and sequencing in chrysanthemum genome, especially in wild chrysanthemum (Fig. 1). On the other hand, the proper protocol for identifying chrysanthemum gene function should be deeply studied. 


\section{Acknowledgments}

This work was supported by Korea Institute of Planning and Evaluation for Technology in Food, Agriculture, Forestry and Fisheries (IPET) through Export Promotion Technology Development Program, funded by Ministry of Agriculture, Food and Rural Affairs (MAFRA) (No. 617076-05-5-SB110).

\section{References}

Coppens F, Wuyts N, Inzé D, Dhondt S (2017) Unlocking the potential of plant phenotyping data through integration and data-driven approaches. Curr. Opin. Syst. Biol. 4:58-63. https://doi.org/10.1016/j.coisb.2017.07.002

Dierck R, Leus L, Dhooghe E, Van Huylenbroeck J, De Riek J, Van Der Straeten D, De Keyser E (2018) Branching gene expression during chrysanthemum axillary bud outgrowth regulated by strigolactone and auxin transport. Plant Growth Regul. 86:23-36. https://doi.org/10.1007/s10725-018-0408-2

Du X, Li W, Sheng L, Deng Y, Wang Y, Zhang W, Yu K, Jiang J, Fang W, et al (2018) Over-expression of chrysanthemum CmDREB6 enhanced tolerance of chrysanthemum to heat stress. BMC Plant Biol. 18:178-178. https://doi.org/10.1186/ s12870-018-1400-8

Fan Q, Song A, Jiang J, Zhang T, Sun H, Wang Y, Chen S, Chen F (2016) CmWRKY1 enhances the dehydration tolerance of chrysanthemum through the regulation of ABA-associated genes. Plos One 11:e0150572-e0150572. https://doi.org/ 10.1371/journal.pone.0150572

Fan Q, Song A, Xin J, Chen S, Jiang J, Wang Y, Li X, Chen F (2015) CmWRKY15 facilitates Alternaria temissima infection of chrysanthemum. Plos One 10:e0143349-e0143349. https://doi.org/10.1371/journal.pone.0143349

Furbank R (2009) Plant phenomics: from gene to form and function. Funct. Plant Biol. 36:V-VI. https://doi.org/10.1071/ FPv36n11_FO

Gao TW, Zhang WW, Song AP, An C, Xin JJ, Jiang JF, Guan ZY, Chen FD, Chen SM (2018a) Phylogenetic and transcriptional analysis of chrysanthemum GRAS transcription factors. Biol. Plant. 62:711-720. https://doi.org/10.1007/s10535-018-0816-1

Gao W, He M, Liu J, Ma X, Zhang Y, Dai S, Zhou Y (2018b) Overexpression of Chrysanthemum lavandulifolium ClCBF1 in Chrysanthemum morifolium 'White Snow' improves the level of salinity and drought tolerance. Plant Physiol. Biochem. 124:50-58. https://doi.org/10.1016/j.plaphy.2018.01.004

Gao Y, Gao Y, Fan M, Yuan L, Wu Z, Zhang Q (2017) Overexpression of Chrysanthemum morifolium SVP gene delays blossoming and regulates inflorescence architecture in transgenic Arabidopsis. Can. J. Plant Sci. 97:1130-1139. https://doi.org/10.1139/cjps-2017-0007

He H, Yajing N, Huawen C, Xingjiao T, Xinli X, Weilun Y, Silan D (2012) cDNA-AFLP analysis of salt-inducible genes expression in Chrysanthemum lavandulifolium under salt treatment. J. Plant Physiol. 169:410-420. https://doi.org/10.1016/ j.jplph.2011.09.013

He H, Yuting L, Ya P, Mi Z, Silan D (2019) Transcriptome analysis of Chrysanthemum lavandulifolium response to salt stress and overexpression a $\mathrm{K}^{+}$transport ClAKT gene-enhanced salt tolerance in transgenic Arabidopsis. J. Amer. Soc. Hort. Sci. 144:219-235. https://doi.org/10.21273/JASHS04629-18

Higuchi Y, Narumi T, Oda A, Nakano Y, Sumitomo K, Fukai S, Hisamatsu T (2013) The gated induction system of a systemic floral inhibitor, antiflorigen, determines obligate short-day flowering in chrysanthemums. Proc. Natl. Acad. Sci. U. S. A. 110:17137. https://doi.org/10.1073/pnas. 1307617110

Hirakawa H, Sumitomo K, Hisamatsu T, Nagano S, Shirasawa K, Higuchi Y, Kusaba M, Koshioka M, Nakano Y, et al (2019) De novo whole-genome assembly in Chrysanthemum seticuspe, a model species of Chrysanthemums, and its application to genetic and gene discovery analysis. DNA Res. 26:195-203. https://doi.org/10.1093/dnares/dsy048

Hong Y, Li M, Dai S (2019) Ectopic expression of multiple Chrysanthemum (Chrysanthemum $\times$ morifolium) R2R3-MYB transcription factor genes regulates anthocyanin accumulation in Tobacco. Periodical Tile 10. https://doi.org/10.3390/genes 10100777

Huang D, Li X, Sun M, Zhang T, Pan H, Cheng T, Wang J, Zhang Q (2016) Identification and characterization of CYC-like genes in regulation of ray floret development in Chrysanthemum morifolium. Front. Plant Sci. 7:1633-1633. https://doi.org/ 10.3389/fpls.2016.01633

Huse SM, Dethlefsen L, Huber JA, Welch DM, Relman DA, Sogin ML (2008) Exploring microbial diversity and taxonomy using SSU rRNA hypervariable tag sequencing. PLOS Genet. 4:e1000255. https://doi.org/10.1371/journal.pgen.1000255

Jaffar MA, Song A, Faheem M, Chen S, Jiang J, Liu C, Fan Q, Chen $\mathrm{F}$ (2016) Involvement of CmWRKY10 in drought tolerance of Chrysanthemum through the ABA-signaling pathway. Int. J. Mol. Sci. 17:693. https://doi.org/10.3390/ijms17050693

Li F, Zhang H, Zhao H, Gao T, Song A, Jiang J, Chen F, Chen S (2018) Chrysanthemum CmHSFA4 gene positively regulates salt stress tolerance in transgenic chrysanthemum. Plant Biotechnol J. 16:1311-1321. https://doi.org/10.1111/pbi.12871

Li H, Chen S, Song A, Wang H, Fang W, Guan Z, Jiang J, Chen F (2014) RNA-Seq derived identification of differential transcription in the chrysanthemum leaf following inoculation with Alternaria tenuissima. BMC Genom. 15:9. https://doi. org/10.1186/1471-2164-15-9

Li P, Song A, Gao C, Jiang J, Chen S, Fang W, Zhang F, Chen F (2015a) The over-expression of a chrysanthemum WRKY transcription factor enhances aphid resistance. Plant Physiol. Biochem. 95:26-34. https://doi.org/10.1016/j.plaphy.2015.07.002

Li P, Song A, Gao C, Wang L, Wang Y, Sun J, Jiang J, Chen F, Chen S (2015b) Chrysanthemum WRKY gene CmWRKY17 negatively regulates salt stress tolerance in transgenic chrysanthemum and Arabidopsis plants. Plant Cell Rep. 34:1365-1378. https://doi.org/10.1007/s00299-015-1793-X

Li S, Li M, Li Z, Zhu Y, Ding H, Fan X, Li F, Wang Z (2019) 
Effects of the silencing of CmMET1 by RNA interference in chrysanthemum (Chrysanthemum morifolium). Plant Biotechnol. Rep. 13:63-72. https://doi.org/10.1007/s11816-019-00516-5 Liang Q-Y, Wu Y-H, Wang K, Bai Z-Y, Liu Q-L, Pan Y-Z, Zhang L, Jiang B-B (2017) Chrysanthemum WRKY gene DgWRKY5 enhances tolerance to salt stress in transgenic chrysanthemum. Sci. Rep. 7:4799-4799. https://doi.org/10.1038/s41598-01705170-x

Liu H, Sun M, Du D, Pan H, Cheng T, Wang J, Zhang Q (2015) Whole-transcriptome analysis of differentially expressed genes in the vegetative buds, floral buds and buds of Chrysanthemum morifolium. Plos One 10:e0128009. https://doi.org/ 10.1371/journal.pone.0128009

Luo C, Chen D, Cheng X, Zhao H, Huang C (2017) Genome size estimations in Chrysanthemum and correlations with molecular phylogenies. Genet. Resour. Crop Evol. 64:1451-1463. https:// doi.org/10.1007/s10722-016-0448-2

Marzachì C, Bosco D (2005) Relative quantification of Chrysanthemum yellows (16Sr I) phytoplasma in its plant and insect host using real-time polymerase chain reaction. Mol. Biotech. 30:117-128. https://doi.org/10.1385/MB:30:2:117

Mochida K, Koda S, Inoue K, Hirayama T, Tanaka S, Nishii R, Melgani F (2018) Computer vision-based phenotyping for improvement of plant productivity: a machine learning perspective. GigaScience 8. https://doi.org/10.1093/gigascience/ giy153

Naing AH, Ai TN, Jeon SM, Park KI, Lim KB, Kim CK (2015) Expression of RsMYB1 in chrysanthemum regulates key anthocyanin biosynthetic genes. Electron. J. Biotechnol. 18: 359-364. https://doi.org/10.1016/j.ejbt.2015.07.001

Nguyen TK, Lim J-H (2019) Tools for Chrysanthemum genetic research and breeding: Is genotyping-by-sequencing (GBS) the best approach? Hortic. Environ. Biotechnol. 60:625-635. https://doi.org/10.1007/s13580-019-00160-6

Nguyen TK, Ha STT, Lim JH (2020) Analysis of chrysanthemum genetic diversity by genotyping-by-sequencing. Hortic. Environ. Biotechnol. 61:903-913. https://doi.org/10.1007/s13580-02000274-2

Nie J, Wen C, Xi L, Lv S, Zhao Q, Kou Y, Ma N, Zhao L, Zhou X (2018) The AP2/ERF transcription factor CmERF053 of chrysanthemum positively regulates shoot branching, lateral root, and drought tolerance. Plant Cell Rep. 37:1049-1060. https://doi.org/10.1007/s00299-018-2290-9

Noda N, Yoshioka S, Kishimoto S, Nakayama M, Douzono M, Tanaka Y, Aida R (2017) Generation of blue chrysanthemums by anthocyanin B-ring hydroxylation and glucosylation and its coloration mechanism. Sci. Adv. 3:e1602785-e1602785. https://doi.org/10.1126/sciadv.1602785

Oda A, Narumi T, Li T, Kando T, Higuchi Y, Sumitomo K, Fukai S, Hisamatsu T (2012) CsFTL3, a chrysanthemum FLOWERING LOCUS T-like gene, is a key regulator of photoperiodic flowering in chrysanthemums. J. Exp. Bot. 63:1461-1477. https://doi.org/10.1093/jxb/err387

Poorter H, Fiorani F, Stitt M, Schurr U, Finck A, Gibon Y, Usadel B, Munns R, Atkin OK, et al (2012) The art of growing plants for experimental purposes: a practical guide for the plant biologist. Funct. Plant Biol. 39:821-838

Puangkrit T, Narumi-Kawasaki T, Takamura T, Fukai S (2018) Isolation and analysis of the key enzyme genes in the flavonoid biosynthesis pathway in chrysanthemum. In, Ed 1208. Intern. Soc. Hortic. Sci. (ISHS), Leuven, Belgium, pp 53-60. https://doi.org/10.17660/ActaHortic.2018.1208.8

Qi S, Yang L, Wen X, Hong Y, Song X, Zhang M, Dai S (2016) Reference gene selection for RT-qPCR analysis of flower development in Chrysanthemum morifolium and Chrysanthemum lavandulifolium. Front. Plant Sci. 7:287-287. https://doi.org/ 10.3389/fpls.2016.00287

Qi Y, Liu Y, Zhang Z, Gao J, Guan Z, Fang W, Chen S, Chen F, Jiang J (2018) The over-expression of a chrysanthemum gene encoding an RNA polymerase II CTD phosphatase-like 1 enzyme enhances tolerance to heat stress. Hortic. Res. 5:37-37. https://doi.org/10.1038/s41438-018-0037-y

Saracco P, Bosco D, Veratti F, Marzachì C (2005) Quantification over time of chrysanthemum yellows phytoplasma (16Sr-I) in leaves and roots of the host plant Chrysanthemum carinatum (Schousboe) following inoculation with its insect vector. Physiol. Mol. Plant Pathol. 67:212-219. https://doi.org/10.1016/ j.pmpp.2006.02.001

Sasaki K, Mitsuda N, Nashima K, Kishimoto K, Katayose Y, Kanamori H, Ohmiya A (2017) Generation of expressed sequence tags for discovery of genes responsible for floral traits of Chrysanthemum morifolium by next-generation sequencing technology. BMC Genom. 18:683. https://doi. org/10.1186/s12864-017-4061-3

Shchennikova AV, Shulga OA, Immink R, Skryabin KG, Angenent GC (2004) Identification and characterization of four Chrysanthemum MADS-Box genes, belonging to the APETALA1/FRUITFULL and SEPALLATA3 subfamilies. Plant Physiol. 134:1632. https://doi.org/10.1104/pp.103.036665

Song A, Gao T, Li P, Chen S, Guan Z, Wu D, Xin J, Fan Q, Zhao $\mathrm{K}$, et al (2016a) Transcriptome-wide identification and expression profiling of the DOF transcription factor gene family in Chrysanthemum morifolium. Front. Plant Sci. 7. https://doi.org/10.3389/fpls.2016.00199

Song A, Gao T, Wu D, Xin J, Chen S, Guan Z, Wang H, Jin L, Chen F (2016b) Transcriptome-wide identification and expression analysis of chrysanthemum SBP-like transcription factors. Plant Physiol. Biochem. 102:10-16. https://doi.org/10.1016/ j.plaphy.2016.02.009

Song A, Wang L, Chen S, Jiang J, Guan Z, Li P, Chen F (2015) Identification of nitrogen starvation-responsive microRNAs in Chrysanthemum nankingense. Plant Physiol. Biochem. 91:41-48. https://doi.org/10.1016/j.plaphy.2015.04.003

Song C, Liu Y, Song A, Dong G, Zhao H, Sun W, Ramakrishnan S, Wang Y, Wang S, et al (2018) The Chrysanthemum nankingense genome provides insights into the evolution and diversification of Chrysanthemum flowers and medicinal traits. Mol. Plant 11:1482-1491. https://doi.org/10.1016/j.molp.2018.10.003

Su J, Jiang J, Zhang F, Liu Y, Ding L, Chen S, Chen F (2019) Current achievements and future prospects in the genetic 
breeding of chrysanthemum: a review. Hortic. Res. 6:109. https://doi.org/10.1038/s41438-019-0193-8

Sun C-H, Yu J-Q, Duan X, Wang J-H, Zhang Q-Y, Gu K-D, Hu D-G, Zheng C-S (2018) The MADS transcription factor CmANR1 positively modulates root system development by directly regulating CmPIN2 in chrysanthemum. Hortic. Res. 5:52-52. https://doi.org/10.1038/s41438-018-0061-y

Takino H, Kitajima S, Hirano S, Oka M, Matsuura T, Ikeda Y, Kojima M, Takebayashi Y, Sakakibara H, et al (2019) Global transcriptome analyses reveal that infection with chrysanthemum stunt viroid (CSVd) affects gene expression profile of chrysanthemum plants, but the genes involved in plant hormone metabolism and signaling may not be silencing target of CSVd-siRNAs. Plant Gene 18:100181. https://doi.org/ 10.1016/j.plgene.2019.100181

Teixeira da Silva JA, Shinoyama H, Aida R, Matsushita Y, Raj SK, Chen F (2013) Chrysanthemum biotechnology: Quo vadis? Crit. Rev. Plant Sci. 32:21-52. https://doi.org/10.1080/07352689. 2012.696461

Tester M, Langridge P (2010) Breeding technologies to increase crop production in a changing world. Science 327:818. https://doi.org/10.1126/science. 1183700

Vallès J, Canela MÁ, Garcia S, Hidalgo O, Pellicer J, SánchezJiménez I, Siljak-Yakovlev S, Vitales D, Garnatje T (2013) Genome size variation and evolution in the family Asteraceae. Caryologia 66:221-235. https://doi.org/10.1080/00087114. 2013.829690

Wang H, Jiang J, Chen S, Fang W, Guan Z, Liao Y, Chen F (2013a) Rapid genomic and transcriptomic alterations induced by wide hybridization: Chrysanthemum nankingense $\times$ Tanacetum vulgare and C. crassum $\times$ Crossostephium chinense (Asteraceae). BMC Genom. 14:902. https://doi.org/10.1186/1471-2164-14-902

Wang H, Jiang J, Chen S, Qi X, Peng H, Li P, Song A, Guan Z, Fang W, et al (2013b) Next-generation sequencing of the Chrysanthemum nankingense (Asteraceae) transcriptome permits large-scale unigene assembly and SSR marker discovery. Plos One 8:e62293-e62293. https://doi.org/10.1371/journal.pone. 0062293

Wang J, Guan Y, Ding L, Li P, Zhao W, Jiang J, Chen S, Chen F (2019a) The CmTCP20 gene regulates petal elongation growth in Chrysanthemum morifolium. Plant Sci. 280:248-257. https://doi.org/10.1016/j.plantsci.2018.12.008

Wang K, Bai Z-Y, Liang Q-Y, Liu Q-L, Zhang L, Pan Y-Z, Liu G-L, Jiang B-B, Zhang F, et al (2018a) Transcriptome analysis of chrysanthemum (Dendranthema grandiflorum) in response to low temperature stress. BMC Genom. 19:319-319. https://doi.org/10.1186/s12864-018-4706-X

Wang K, Wu Y-H, Tian X-Q, Bai Z-Y, Liang Q-Y, Liu Q-L, Pan Y-Z, Zhang L, Jiang B-B (2017) Overexpression of DgWRKY4 enhances salt tolerance in Chrysanthemum seedlings. Front. Plant Sci. 8:1592-1592. https://doi.org/10.3389/fpls.2017.01592 Wang S, Song Q, Li S, Hu Z, Dong G, Song C, Huang H, Liu Y (2018b) Assembly of a complete mitogenome of Chrysanthemum nankingense using Oxford nanopore long reads and the diversity and evolution of Asteraceae mitogenomes. Genes (Basel) 9. https://doi.org/10.3390/genes9110547

Wang S, Zhang C, Zhao J, Li R, Lv J (2019b) Expression analysis of four pseudo-response regulator (PRR) genes in Chrysanthemum morifolium under different photoperiods. PeerJ 7:e6420. https://doi.org/10.7717/peerj.6420

Wang Y, Huang H, Ma Y-P, Jianxin F, Wang L, Dai S (2014) Construction and de novo characterization of a transcriptome of Chrysanthemum lavandulifolium: Analysis of gene expression patterns in floral bud emergence. Plant Cell 116. https://doi. org/10.1007/s11240-013-0404-1

Wei Q, Ma C, Xu Y, Wang T, Chen Y, Lü J, Zhang L, Jiang C-Z, Hong B, et al (2017) Control of chrysanthemum flowering through integration with an aging pathway. Nat. Commun. 8:829-829. https://doi.org/10.1038/s41467-017-00812-0

Xia Y, Hu Z, Li X, Wang P, Zhang X, Li Q, Lu C (2016) The complete chloroplast genome sequence of Chrysanthemum indicum. Mitochondrial DNA Part A 27:4668-4669. https:// doi.org/10.3109/19401736.2015.1106494

Xu Y, Gao S, Yang Y, Huang M, Cheng L, Wei Q, Fei Z, Gao J, Hong B (2013) Transcriptome sequencing and whole genome expression profiling of chrysanthemum under dehydration stress. BMC Genom. 14:662. https://doi.org/10.1186/14712164-14-662

Yagi M (2018) Recent progress in whole genome sequencing, high-density linkage maps, and genomic databases of ornamental plants. Breed. Sci. 68:62-70. https://doi.org/10.1270/jsbbs. 17080

Yang Y, Ma C, Xu Y, Wei Q, Imtiaz M, Lan H, Gao S, Cheng L, Wang $M$, et al (2014) A Zinc finger protein regulates flowering time and abiotic stress tolerance in Chrysanthemum by modulating gibberellin biosynthesis. The Plant Cell 26: 2038-2054. https://doi.org/10.1105/tpc.114.124867

Yeong TJ, Pin Jern K, Yao LK, Hannan MA, Hoon STG (2019) Applications of photonics in agriculture sector: A review. Molecules (Basel, Switzerland) 24:2025. https://doi.org/10.3390/ molecules 24102025

Yue J, Zhu C, Zhou Y, Niu X, Miao M, Tang X, Chen F, Zhao W, Liu Y (2018) Transcriptome analysis of differentially expressed unigenes involved in flavonoid biosynthesis during flower development of Chrysanthemum morifolium 'Chuju'. Sci. Rep. 8:13414-13414. https://doi.org/10.1038/s41598-018-31831-6

Zhang F, Dong W, Huang L, Song A, Wang H, Fang W, Chen F, Teng N (2015) Identification of microRNAs and their targets associated with embryo abortion during Chrysanthemum cross breeding via high-throughput sequencing. Plos One 10:e0124371. https://doi.org/10.1371/journal.pone.0124371

Zhang F, Zhao J, Xu S, Fang W, Chen F, Teng N (2017) MicroRNA and putative target discoveries in Chrysanthemum polyploidy breeding. Int. J. Genomics 2017:6790478-6790478. https://doi. org/10.1155/2017/6790478 